\title{
Lingmao Formula Combined with Entecavir for HBeAg-Positive Chronic Hepatitis B Patients with Mildly Elevated Alanine Aminotransferase: A Multicenter, Randomized, Double-Blind, Placebo-Controlled Trial
}

\author{
Xiao-Jun Zhu, ${ }^{1,2}$ Xue-Hua Sun, ${ }^{1,2}$ Zheng-Hua Zhou, ${ }^{1,2}$ Shun-Qing Liu, ${ }^{1,2}$ \\ Hua $\mathrm{Lv},{ }^{3}$ Man Li, ${ }^{4} \mathrm{Lu} \mathrm{Li},{ }^{1,2}$ and Yue-Qiu Gao ${ }^{1,2,4}$ \\ ${ }^{1}$ Department of Hepatopathy, Shuguang Hospital, Shanghai University of Traditional Chinese Medicine, \\ 528 Zhangheng Road, Shanghai 201203, China \\ ${ }^{2}$ Shanghai Traditional Chinese Medicine Clinical Center of Hepatopathy, 528 Zhangheng Road, Shanghai 201203, China \\ ${ }^{3}$ Center for Clinical Effect Evaluation, Shuguang Hospital, Shanghai University of Traditional Chinese Medicine, \\ 528 Zhangheng Road, Shanghai 201203, China \\ ${ }^{4}$ Shanghai Key Laboratory of Traditional Chinese Medicine, 528 Zhangheng Road, Shanghai 201203, China
}

Correspondence should be addressed to Yue-Qiu Gao; gaoyueqiu@hotmail.com

Received 31 March 2013; Revised 20 July 2013; Accepted 21 July 2013

Academic Editor: Lixing Lao

Copyright (C) 2013 Xiao-Jun Zhu et al. This is an open access article distributed under the Creative Commons Attribution License, which permits unrestricted use, distribution, and reproduction in any medium, provided the original work is properly cited.

\begin{abstract}
Objective. To determine the efficacy and safety of Lingmao Formula combined with entecavir for HBeAg-positive chronic hepatitis B patients with mildly elevated alanine aminotransferase (ALT). Methods. 301 patients were randomly assigned to receive Lingmao Formula combined with entecavir (treatment group) or placebo combined with entecavir (control group) for 52 weeks. The outcomes of interest included the reduction of serum HBV DNA level, HBeAg loss, HBeAg seroconversion, ALT normalization, and histological improvement. Results. The mean decrease of serum HBV DNA level from baseline and the percentage of patients who had reduction in serum HBV DNA level $\geq 2 \lg$ copies $/ \mathrm{mL}$ in treatment group were significantly greater than that in control group (5.5 versus $5.4 \mathrm{lg}$ copies $/ \mathrm{mL}, P=0.010 ; 98.5 \%$ versus $92.6 \%, P=0.019$ ). The percentage of $\mathrm{HBeAg}$ loss in treatment group was $22.8 \%$, which was much higher than a percentage of $12.6 \%$ in control group $(P=0.038)$. There was no significant difference between the two groups in histological improvement. Safety was similar in the two groups. Conclusions. The combination of Lingmao Formula with entecavir could result in significant decrease of serum HBV DNA and increase of HBeAg loss for HBeAg-positive chronic hepatitis B patients with mildly elevated ALT without any serious adverse events. Clinical trial registration number is ChiCTR-TRC-09000594.
\end{abstract}

\section{Introduction}

Hepatitis B virus (HBV) is the most common pathogen of chronic liver diseases. According to the data released by World Health Organization (WHO), two billion people are estimated to have been infected with HBV worldwide and 350 million are chronic HBV carriers [1]. HBV-related liver diseases, including hepatitis, cirrhosis, and hepatocellular carcinoma, are responsible for more than one million deaths annually [2]. Recently, an nationwide survey conducted in China reported a HBV infection rate of $7.18 \%$ in Chinese population [3, 4]. Accordingly, about 93 million HBV carriers and 20 million patients with chronic hepatitis $\mathrm{B}(\mathrm{CHB})$ are estimated in China [5]. Even worse, more than 300,000 Chinese patients died from the HBV-related diseases every year [6].

Current guidelines for the management of $\mathrm{CHB}$ recommend that the antiviral treatment should be initiated for hepatitis $\mathrm{B}$ e antigen $(\mathrm{HBeAg})$ positive patients if the $\mathrm{HBV}$ DNA levels are $\geq 1 \times 10^{5}$ copies $/ \mathrm{mL}$ and the ALT levels are at least 2-folds than the upper limit of normal (ULN) [7-10]. HBV-infected patients with mildly elevated serum ALT levels 
$(1-2 \times \mathrm{ULN})$ usually show significant necroinflammation and fibrosis when liver biopsy performed [11]. These patients are at similar risk to develop severe complications as compared with those having higher serum ALT levels [12]. EASL (European Association for the Study of the Liver) Clinical Practice Guidelines suggest that patients with mildly elevated serum ALT levels should also be treated when the HBV DNA levels are higher than $1 \times 10^{5}$ copies/mL and liver biopsy disclosed moderate or severe active necroinflammation and/or fibrosis using a standardized scoring system [10]. However, a few studies have demonstrated that HBeAg-positive $\mathrm{CHB}$ patients with mildly elevated ALT respond poorly to the treatment with lamivudine, entecavir, or interferon [13-15]. Therefore, it is urgent to develop novel agents or therapy strategies for this specific patient population.

In China, several traditional Chinese herbs have been used to treat $\mathrm{CHB}$ for hundreds of decades. Recently, contemporary clinical researches of traditional Chinese medicines for $\mathrm{CHB}$ were reviewed. The results showed that some traditional Chinese herbs may enhance the antiviral activities of interferon and lamivudine and improve the liver function of $\mathrm{CHB}$ patients [16]. The therapeutic effects of a few of Chinese herbs for CHB patients, such as astragalus, polygonum cuspidatum, and phyllanthus urinaria, have been identified through inhibiting HBV replication [17-19]. The Lingmao Formula, an empirical formula widely prescribed in traditional Chinese medicine for $\mathrm{CHB}$ patients, has showed promising inhibition activity of $\mathrm{HBsAg}$ and $\mathrm{HBeAg}$ secretions in vitro in our previous study [20]. However, no additional data regarding the efficacy and safety of the Lingmao Formula is available until now. To determine the therapeutic role of the Lingmao Formula for $\mathrm{CHB}$ patients, especially for the $\mathrm{CHB}$ patients with mildly evaluated serum ALT levels, a multicenter, randomized, double-blind, and placebo-controlled clinical trial was carried out in the present study. Entecavir was used as a positive control agent and the Lingmao Formula was administrated in combination with entecavir for 52 weeks. The efficacy and safety of the Lingmao Formula combined with entecavir were thoroughly evaluated.

\section{Materials and Methods}

2.1. Study Design. This study was a multicenter, randomized, double-blind, and placebo-controlled clinical trial to evaluate the efficacy and safety of the Lingmao Formula combined with entecavir for HBeAg-positive chronic hepatitis B patients with mildly elevated alanine aminotransferase (ALT). All the patients were treated with entecavir $0.5 \mathrm{mg}$ daily and were randomly assigned to receive the Lingmao Formula or placebo ( $4.5 \mathrm{~g}$ twice daily, oral administration) for 52 weeks, respectively, in treatment and control groups. The Lingmao Formula was composed of Yinyanghuo (Epimedium brevicornum Maxim. leaf), Huangqi (Astragalus membranaceus (Fisch.) Bge. Var. mongholicus (Bge.) Hsiao. root), Huhuanglian (Picrorhiza scrophulariiflora Pennell root), Maozhaocao (Ranunculus ternatus Thunb root), Nvzhenzi (Ligustrum lucidum Ait. ripe fruit), and
Qingpi (Citrus reticulate Blanco immature fruit peel). The concentrated granules of the six herbs provided by Jiang Yin Tianjiang Pharmaceutical Co. Ltd. were used in this study. The concentrated granules of the six herbs were packaged individually according to the prescription of the Lingmao Formula, and they were mixed and dissolved by warm water when taken orally. Weights of the six herb concentrated granules in the Lingmao Formula ( $4.5 \mathrm{~g}$ ) are Yinyanghuo $0.375 \mathrm{~g}$ (lot number: 0903067, amount to original herb of $7.5 \mathrm{~g}$ ), Huangqi $1.125 \mathrm{~g}$ (lot number: 0812028, amount to original herb of $7.5 \mathrm{~g}$ ), Huhuanglian $0.75 \mathrm{~g}$ (lot number: 0904046, amount to original herb of $4.5 \mathrm{~g}$ ), Maozhaocao $0.75 \mathrm{~g}$ (lot number: 0802045, amount to original herb of $7.5 \mathrm{~g}$ ), Nvzhenzi $0.75 \mathrm{~g}$ (lot number: 0812091, amount to original herb of 7.5 g), and Qingpi $0.75 \mathrm{~g}$ (lot number: 0811006, amount to original herb of $4.5 \mathrm{~g}$ ).

There is a corresponding placebo also in concentrated granule form for each herb in the Lingmao Formula. The placebos were provided by the same company: placebo of Yinyanghuo (lot number: 0903334), placebo of Huangqi (lot number: 0903332), placebo of Huhuanglian (lot number: 0903336), placebo of Maozhaocao (lot number: 0903335) placebo of Nvzhenzi (lot number: 0903333), and placebo of Qingpi (lot number: 0903337). They were composed of food coloring (tartrazine, sunset yellow, and caramel color), bittering agent (sucrose octaacetate), and pharmaceutical excipient (Lactose). Food coloring, bittering agent, and pharmaceutical excipient were conducted in accordance with the Chinese regulation on the management of pharmaceutical excipients and Chinese hygienic standards for uses of food additives. The placebos shared the same package and label as the herb granules. The appearance, taste and solubitity of placebos were similar to the herb granules. They were also taken the same way as the Lingmao Formula. This study was conducted in accordance with the ethics principles of the Declaration of Helsinki and regulation of clinical trials [21]. It was also approved by the Ethical Committee of Shuguang Hospital, Shanghai University of Traditional Chinese Medicine. Written informed consent was obtained from all enrolled patients.

\subsection{Sample Size Calculation and Randomization. For HBe-} Ag-positive patients with mildly elevated ALT, our previous pilot study showed that HBV DNA was undetectable by PCR assay (HBV DNA $<1000$ copies $/ \mathrm{mL}$ ) in $47 \%$ of patients when treated by entecavir alone and in $65 \%$ of patients treated by both the Lingmao Formula and entecavir. If $\alpha=0.05, \beta=$ 0.20 , and a drop-out rate of $20 \%$ was assumed, 300 subjects would be required for enrollment into the study. By using a block randomization method, the enrolled patients were assigned into two groups with four patients in one block with a proportion of $1: 1$. The randomization was performed by SAS version 9.0 (SAS Institute, Cary, NC, USA).

2.3. Study Population. The inclusion criteria included aged from 18 to 65; hepatitis B surface antigen (HBsAg) was 
detectable for at least 24 weeks; HBeAg-positive; compensated liver function; mildly elevated serum ALT levels (1$2 \times \mathrm{ULN}$ ) at least 4 weeks before screening; HBV DNA level was $\geq 1 \times 10^{5}$ copies/mL by PCR assay at least 4 weeks before screening; untreated with any nucleoside analogues, pegylated interferon $\alpha$, or interferon $\alpha$ at least 12 months before screening; and liver-biopsy specimens with a Scheuer necroinflammatory grade of 2 or greater within 12 weeks before randomization. The exclusion criteria included being coinfected with HAV, HCV, HDV, HEV, and HIV; hepatocellular carcinoma; other forms of liver disease; being other severe primary disease or mental disorder disease; treated to decrease the ALT level within three months; and pregnant and lactating women. The rejection and withdrawal criteria were misincluded or misdiagnosed; without any test record; did not finish the trial according to the program; abnormal serum ALT not due to CHB.

2.4. Efficacy Endpoints. The primary endpoints were listed as follows (1) Virological response: HBV DNA was quantified by real-time PCR assay and the lowest limit of quantification was set at $1 \times 10^{3}$ copies $/ \mathrm{mL}$. The extent of HBV DNA change and the rate of decrease in serum HBV DNA of $>2 \lg$ copies/mLwere computed. (2) Serological response: the loss rates of $\mathrm{HBeAg}$ and $\mathrm{HBsAg}$ or the seroconversion rates of $\mathrm{HBeAg}$ and $\mathrm{HBsAg}$ were, respectively, calculated. (3) Biochemical response: the rate of ALT normalization between the two groups was analyzed. (4) Histological response: positive response was defined as that necroinflammatory was improved by at least one grade in the Scheuer necroinflammatory grade with no worsening in the fibrosis stage, or fibrosis was improved by at least one grade in the Scheuer fibrosis stage with no worsening in the necroinflammatory grade.

2.5. Resistance and Safety Measurements. A resistance analysis was undertaken through identifying the HBV polymerase substitutions which were associated with reduced susceptibility to entecavir. Serum samples from patients at baseline, patients with viral breakthrough, and patients having more than $1 \times 10^{3}$ copies/mL of HBV DNA at week 52 were obtained and analyzed. HBV DNA was quantified by using real-time PCR method and the genotype of HBV DNA polymerase was sequenced.

Patients were advised to report any change in health. All adverse events were documented by the physicians. Electrocardiogram, complete blood count, urine analysis, serum creatinine, and urea nitrogen were also performed before enrollment and at week 52. The primary safety endpoint was the proportion of patients who discontinued the study medication because of clinical or laboratory-determined adverse events. Other safety evaluations included analyses of adverse events, serious events, and death.

2.6. Statistical Analysis. Statistical analyses were performed using SPSS statistics software (version 17.0, IBM Inc., NY, USA). Continuous variables were described by means \pm standard deviation (SD). Comparison of continuous variables
TABLE 1: Demographic and baseline characteristics of the patients.

\begin{tabular}{|c|c|c|c|}
\hline Characteristics & $\begin{array}{c}\text { Treatment } \\
\text { group } \\
(n=136)\end{array}$ & $\begin{array}{c}\text { Control } \\
\text { group } \\
(n=135)\end{array}$ & $P$ value \\
\hline Age $(\text { years })^{\dagger}$ & $33.5 \pm 10.1$ & $32.8 \pm 9.1$ & 0.346 \\
\hline Male & $104(76.5)$ & $104(77.0)$ & 1.000 \\
\hline Height $(\mathrm{cm})^{\dagger}$ & $169.1 \pm 6.6$ & $168.9 \pm 6.1$ & 0.516 \\
\hline Weight $(\mathrm{kg})^{\dagger}$ & $65.0 \pm 10.0$ & $65.5 \pm 10.7$ & 0.824 \\
\hline Body mass index $\left(\mathrm{kg} / \mathrm{m}^{2}\right)^{\dagger}$ & $22.7 \pm 2.8$ & $22.9 \pm 3.0$ & 0.775 \\
\hline Course of illness (years) ${ }^{\dagger}$ & $7.7 \pm 6.1$ & $6.9 \pm 5.8$ & 0.450 \\
\hline HBV DNA (lgcopies/mL) ${ }^{\dagger}$ & $7.2 \pm 1.2$ & $7.3 \pm 1.4$ & 0.456 \\
\hline $\begin{array}{l}\text { Grade of } \\
\text { necroinflammatory* }\end{array}$ & & & 0.600 \\
\hline G2 & $28(68.3)$ & $29(74.4)$ & \\
\hline G3 & $10(24.4)$ & $9(23.1)$ & \\
\hline G4 & $3(7.3)$ & $1(2.6)$ & \\
\hline Stage of fibrosis* & & & 0.621 \\
\hline So & $0(0.0)$ & $1(2.6)$ & \\
\hline $\mathrm{S} 1$ & $12(29.3)$ & $7(17.9)$ & \\
\hline S2 & $17(41.5)$ & $19(48.7)$ & \\
\hline S3 & $9(22.0)$ & $10(25.6)$ & \\
\hline S4 & $3(7.3)$ & $2(5.1)$ & \\
\hline $\begin{array}{l}\text { Prior resistance by } \\
\text { genotypic analysis* }\end{array}$ & $0(0)$ & $0(0)$ & 1.000 \\
\hline
\end{tabular}

${ }^{*}$ A total of 80 patients had adequate baseline and liver-biopsy specimens at week 52 (41 patients in treatment group and 39 in control group). Values in parentheses are percentages unless indicated otherwise; ${ }^{\dagger}$ value was presented as mean \pm standard deviation.

was analyzed by $t$-test. Categorical variables were described by rate and percentage. Comparison of categorical variables was conducted by Pearson's chi-square test or Fisher's exact test. All $P$ values were two-sided with significance level at 0.05 .

\section{Results}

3.1. Study Population. From December 2008 to March 2011, a total of 363 patients were recruited from 12 Chinese hospitals. After 62 patients were excluded, 301 eligible patients were randomly assigned into treatment group or control group in a double-blinded manner. One hundred and fifty patients were treated with the Lingmao Formula and entecavir, and the other 151 patients were treated with placebo and entecavir. In a double-blinded manner, 271 patients were received (136 in the treatment group and 135 in the control group). Among them, 41 patients in the treatment group and 39 patients in the control group had adequate liver biopsy specimens for histopathological evaluation at both baseline and trial end. The baseline characteristics of these patients in the two groups are shown in Table 1 and no difference were found between the two groups. Flow diagram of the trial is show in Figure 1. 
TABLE 2: Histological, virologic, serologic, and biochemical response at week 52.

\begin{tabular}{|c|c|c|c|}
\hline End points & Treatment group $(n=136)$ & Control group $(n=135)$ & $P$ value \\
\hline Necroinflammatory improvement ${ }^{*}$ & $22(53.7)$ & $13(33.3)$ & 0.076 \\
\hline Fibrosis improvement* & $17(41.5)$ & $14(35.9)$ & 0.652 \\
\hline HBV DNA $<1000$ copies/mL by PCR assay & $93(68.4)$ & $91(67.4)$ & 0.897 \\
\hline$\geq 2 \lg$ copies/mL reduction in HBV DNA compared to baseline & $134(98.5)$ & $125(92.6)$ & 0.019 \\
\hline Mean change in HBV DNA from baseline by PCR assay $(\lg \text { copies } / \mathrm{mL})^{\dagger}$ & $5.5 \pm 1.6$ & $5.4 \pm 1.9$ & 0.010 \\
\hline HBeAg loss & $31(22.8)$ & $17(12.6)$ & 0.038 \\
\hline Undetectable HBV DNA and HBeAg loss & $29(21.3)$ & $12(8.9)$ & 0.006 \\
\hline HBeAg seroconversion & $19(14.0)$ & $15(11.1)$ & 0.583 \\
\hline Undetectable HBV DNA and $\mathrm{HBe} A g$ seroconversion & $19(14.0)$ & $11(8.1)$ & 0.175 \\
\hline HBsAg loss & $2(1.5)$ & $1(0.7)$ & 1.000 \\
\hline ALT normalization & $106(77.9)$ & $106(78.5)$ & 1.000 \\
\hline
\end{tabular}

${ }^{*}$ A total of 80 patients had adequate baseline and liver-biopsy specimens at week 52 (41 patients in treatment group and 39 in control group). Values in parentheses are percentages unless indicated otherwise; ${ }^{\dagger}$ value was presented as mean \pm standard deviation.

TABlE 3: Accumulative adverse events during the 52 weeks of treatment.

\begin{tabular}{lccc}
\hline Adverse event & $\begin{array}{c}\text { Treatment group } \\
(n=136)\end{array}$ & $\begin{array}{c}\text { Control group } \\
(n=135)\end{array}$ & $P$ value \\
\hline Fatigue & $28(20.6)$ & $25(18.5)$ & 0.760 \\
Upper abdominal & $21(15.4)$ & $19(14.1)$ & 0.864 \\
pain & $24(17.6)$ & $22(16.3)$ & 0.872 \\
Diarrhea & $21(15.4)$ & $22(16.3)$ & 0.869 \\
Nausea & $33(24.3)$ & $35(25.9)$ & 0.781 \\
Dyspepsia & $12(8.8)$ & $15(11.1)$ & 0.550 \\
Dizziness & $24(17.6)$ & $29(21.5)$ & 0.447 \\
Itching & $20(14.7)$ & $16(11.9)$ & 0.592 \\
Headache & $8(5.9)$ & $9(6.7)$ & 0.808 \\
Cough & $0(0)$ & $0(0)$ & \\
Serious adverse & & $1(0.7)$ & 1.000 \\
event & $2(1.4)$ & $0(0)$ & \\
Discontinuation due & & & \\
to adverse event & $0(0)$ & \\
Death &
\end{tabular}

Values in parentheses are percentages unless indicated otherwise.

3.2. Virologic Response. As shown in Table 2, the mean decrease of serum HBV DNA level from baseline in treatment group was significantly greater than that in control group (5.5 versus $5.4 \mathrm{lg}$ copies $/ \mathrm{mL}, P=0.010$ ) at week 52. Also, the percentage of reduction in serum HBV DNA level $\geq 2 \mathrm{lg}$ copies/mL in treatment group was significantly higher than that in control group (98.5\% versus $92.6 \%, P=0.019)$. HBV DNA was undetectable by PCR assay in $68.4 \%$ of patients in the treatment group at week 52 and no difference was found when compared to that in the control group $(67.4 \%$, $P=0.897)$.

3.3. Serologic Response. As listed in Table 2, the rate of $\mathrm{HBeAg}$ loss in treatment group was $22.8 \%$, which was much higher than the rate of $12.6 \%$ in control group $(P=0.038)$. At week 52, both undetectable HBV DNA and HBeAg loss were identified in 29 patients of the treatment group (21.3\%) and 12 of the control group $(8.9 \%, P=0.006)$. However, the rates of $\mathrm{HBeAg}$ seroconversion between the two groups are not significantly different $(14.0 \%$ versus $11.1 \%, P=$ 0.583). At week 52, both undetectable HBV DNA and HBeAg seroconversion were identified in 19 patients of the treatment group (14.0\%) and 11 of the control group (8.1\%, $P=0.175)$. HBsAg loss only occurred in two patients in treatment group $(1.5 \%)$ and only one in control group $(0.7 \%)$.

3.4. Biochemical Response. The percentage of ALT normalization in the treatment group was slightly lower than that in the control group and no significant difference was found between them $(77.9 \%$ versus $78.5 \%, P=1.000$; Table 2$)$.

3.5. Histological Response. The histopathological evaluation showed that $53.7 \%$ of patients in treatment group and $33.3 \%$ of patients in control group achieved an improved necroinflammatory ( $P=0.076$; Table 2$)$. Similarly, $41.5 \%$ of patients in the treatment group and $35.9 \%$ of patients in the control group underwent an improved fibrosis $(P=0.652$, Table 2$)$.

3.6. Resistance. No patient in the study had virologic rebound (an increase of HBV DNA levels from nadir of $\geq 1 \mathrm{lg}$ copies/mL by PCR assay) during the trial. Genotypic resistance occurred in only one patient in control group at week 52, who had mutations in the L180 M, M250V, and M204I. Elevation in alanine aminotransferase was not observed in this patient.

3.7. Safety and Adverse Events. As shown in Table 3, the frequency of adverse events during the whole trial was similar between the two groups. The most frequent adverse events were upper abdominal pain, diarrhea, fatigue, nausea, dyspepsia, dizziness, itching, headache, and cough, and most of them were of mild grade. There was no serious adverse event in the two groups. In treatment group, one patient discontinued because of an increase in ALT and the other patient discontinued because of mild diarrhea. In control 


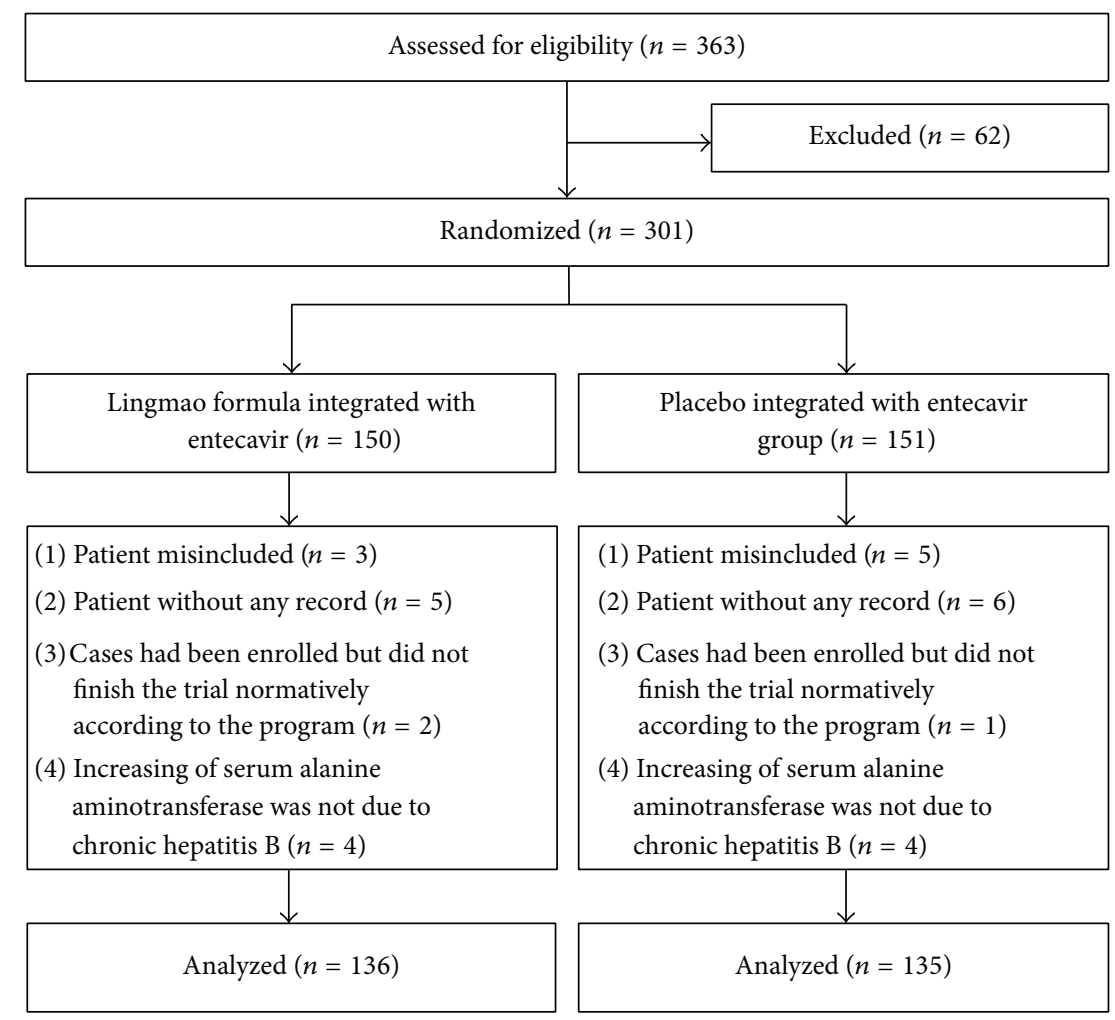

FIGURE 1: Flow diagram of the randomized clinical trial.

group, one patient also discontinued due to an increase in ALT. None of patients in the two groups underwent hepatic decompensation. Additionally, there were no significant changes in electrocardiogram, complete blood count, urine analysis, serum creatinine, and urea nitrogen prior and after drug administration.

\section{Discussion}

Current management guidelines for $\mathrm{CHB}$ patients with high viral load emphasize that antiviral treatment should be initiated once serum ALT exceeds $2 \times$ ULN. But for patients with a mildly elevated ALT, antiviral treatment is recommended to be initiated only in patients with biopsy-confirmed moderate/severe inflammation or significant fibrosis [7-10]. Previous studies have demonstrated that significant pathological changes also occur though ALT is less than $2 \times \mathrm{ULN}$ $[11,22,23]$. Our study showed that $83.3 \%$ of the Chinese HBeAg-positive CHB patients with mildly elevated ALT had moderate or severe active necroinflammation [24]. There is an emerging opinion that the original definition of normal or healthy ALT is too high $[25,26]$. It is possible that stringent adherence to the serum ALT threshold of $2 \times$ ULN could exclude a proportion of patients who might benefit from the antiviral treatment. Therefore, ALT is not reliable and biopsy is necessary for the patients with serum ALT levels between 1 and $2 \times$ ULN.

In our study, the Lingmao Formula combined with entecavir shows significant superiority to placebo combined with entecavir in terms of the rate of decrease in serum HBV DNA of $>2 \lg$ copies $/ \mathrm{mL}$ and the mean reduction of HBV DNA. Similarly, the combination administration was superior to entecavir alone in HBeAg loss. The rate of $\mathrm{HBeAg}$ loss increased by $10.2 \%$ in treatment group compared to control group. Taken together, the Lingmao Formula enhances the antiviral activity of entecavir.

Arresting or reversing liver damage is a principal goal in the management of $\mathrm{CHB}$. Histopathological evaluation of the liver tissue remains the definitive method of assessing the disease progression or the drug response for $\mathrm{CHB}$ patients. In this study, part of the enrolled patients underwent histopathological evaluation concerning their live damage prior and after trial. The results showed that the Lingmao Formula integrated with entecavir could improve the necroinflammatory and fibrosis for $\mathrm{CHB}$ patients though no significant difference was detected between the two groups. Particularly, the improvement of necroinflammatory in the treatment group was higher than that in the placebo integrated with entecavir group by nearly $20 \%$. More sample size of biopsy specimens in further studies is warranted to clarify the superiority of the Lingmao Formula combined with entecavir for $\mathrm{CHB}$ patients.

For HBeAg-positive patients with mildly elevated ALT, entecavir shows lower response in histological improvement, loss of serum HBV DNA, and HBeAg seroconversion than for those with ALT greater than $2 \times$ ULN [14]. When HBeAgpositive patients with mildly elevated ALT receive antiviral therapy, they usually need longer treatment period than 
those with ALT levels greater than $2 \times$ ULN. The longer treatment period means the increase of the care cost. The economic burden of CHB on national healthcare systems is even more pronounced in China. $\mathrm{Li}$ and colleagues reported that $\mathrm{CHB}$ accounted for about $4 \%$ of the national healthcare expenditure in Hong Kong [27]. The endpoints of antiviral treatment for $\mathrm{HBeAg}$-positive patients are disappearance of serum HBV DNA and either HBeAg loss or HBeAg seroconversion. The undetectable HBV DNA and HBeAg loss increased by $12.4 \%$ in treatment group compared to control group. Obviously, the integrative treatment might bring a more optimal cost-effectiveness ratio than entecavir alone for CHB patients.

Longer treatment period also means the increased incidence of drug resistance. Preliminary data suggest that the rate of entecavir resistance remained at $1.2 \%$ in nucleosidenaive patients after up to 5 years of treatment [28]. In the present study, one patient was found to represent resistant genotype in entecavir group. No viral resistance was observed in the Lingmao Formula with entecavir group at week 52. The potential for reducing the rate of drug resistance in the Lingmao Formula with entecavir group should be monitored for longer time.

When the safety is concerned, the Lingmao Formula integrated with entecavir showed similar tolerability profile with placebo combined with entecavir. A 52-week continued treatment of the Lingmao Formula integrated with entecavir is well tolerated for the HBeAg-positive patients with mildly elevated ALT.

\section{Conclusions}

This study showed that the Lingmao Formula combined with entecavir had a significant antiviral priority to entecavir alone for $\mathrm{HBeAg}$-positive $\mathrm{CHB}$ patients with mildly elevated ALT. Together with its safety profile, our data suggests that the Lingmao Formula integrated with entecavir could be considered as an alternative therapy for CHB patients.

\section{Conflict of Interests}

The authors declared no conflict of interests.

\section{Authors' Contribution}

Xiao-Jun Zhu and Xue-Hua Sun contributed equally to this work.

\section{Acknowledgments}

This study was supported by 12 hospitals in China. The authors thank the researchers: Xiao-Rong Chen and BoZong Tang (Shanghai Public Health Clinical Center); Wei Zhang and Hai-Yan Zhang (Longhua Hospital affiliated to Shanghai University of Traditional Chinese Medicine); Peng Guo (Xiyuan Hospital of China Academy of Chinese Medical Sciences); Xiao-Yun Hu and Lin-Hui Xiao (Sichuan Province Traditional Chinese Medicine Hospital); Yong Li
(Shandong Province Traditional Chinese Medicine Hospital); Wei-Bing Shi and Chang-Lin Peng (Anhui Province Traditional Chinese Medicine Hospital); Rui-Min Liang and Xiao-xia (Guo Shanxi Province Traditional Chinese Medicine Hospital); Xiao-Hong Zhu and Jie Hu (Zhejiang Province Traditional Chinese Medicine Hospital); Xiao-Ling Chi and Huan-Ming Xiao (Guangdong Province Traditional Chinese Medical Hospital). This work was supported by the National Natural Science Foundation of China (81072792 and 81102570), Science Research Project of Eleven Fiveyear Plan (no. 2008ZX10005-006), Program of Shanghai Subject Chief Scientist (no. 10XD1404100), Leading Academic Discipline Project of Shanghai Municipal Education Commission (no. J50307), and Innovative Research Team in Universities, Shanghai Municipal Education Commission, Shanghai Natural Science Foundation (no. 10ZR1430900).

\section{References}

[1] D. Lavanchy, "Hepatitis B virus epidemiology, disease burden, treatment, arid current and emerging prevention and control measures," Journal of Viral Hepatitis, vol. 11, no. 2, pp. 97-107, 2004.

[2] D. Ganem and A. M. Prince, "Hepatitis B virus infectionnatural history and clini-cal consequences," New England Journal of Medicine, vol. 350, no. 11, pp. 1118-1129, 2004.

[3] J. Liu and D. Fan, "Hepatitis B in China," The Lancet, vol. 369, no. 9573, pp. 1582-1583, 2007.

[4] X. Liang, S. Bi, W. Yang et al., "Epidemiological serosurvey of Hepatitis B in China-Declining HBV prevalence due to Hepatitis B vaccination," Vaccine, vol. 27, no. 47, pp. 6550-6557, 2009.

[5] F. M. Lu and H. Zhuang, "Management of hepatitis B in China," Chinese Medical Journal (English Edition), vol. 122, no. 1, pp. 3-4, 2009.

[6] J.-D. Jia and H. Zhuang, "The overview of the seminar on chronic hepatitis B," Chinese Journal of Hepatology, vol. 12, no. 11, pp. 698-699, 2004.

[7] A. S. F. Lok and B. J. McMahon, "Chronic hepatitis B: update 2009," Hepatology, vol. 50, no. 3, pp. 661-662, 2009.

[8] Chinese Society of Hepatology and Chinese Society of Infectious Disease, Chinese Medical Association, "The guideline of prevention and treatment for chronic hepatitis B (2010)," Chinese Journal of Gastroenterology and Hepatology, vol. 20, no. 2, pp. 1-14, 2011 (Chinese).

[9] Y. F. Liaw, J. H. Kao, T. Piratvisuth et al., "Asian-Pacific consensus statement on the management of chronic hepatitis B: a 2012 update," Hepatology International, vol. 6, no. 3, pp. 531561,2012

[10] European Association for the Study of the Liver, "EASL Clinical Practice Gui-delines: management of chronic hepatitis B," Hepatology, vol. 57, no. 1, pp. 167-185, 2012.

[11] P. S. Tsang, H. Trinh, R. T. Garcia et al., "Significant prevalence of histologic disease in patients with chronic hepatitis B and mildly elevated serum alanine amino-transferase levels," Clinical Gastroenterology and Hepatology, vol. 6, no. 5, pp. 569-574, 2008.

[12] M.-F. Yuen, H.-J. Yuan, D. K.-H. Wong et al., "Prognostic determinants for chronic hepatitis B in Asians: therapeutic implications," Gut, vol. 54, no. 11, pp. 1610-1614, 2005. 
[13] R. P. Perrillo, C.-L. Lai, Y.-F. Liaw et al., "Predictors of HBeAg loss after lamivudine treatment for chronic hepatitis B," Hepatology, vol. 36, no. 1, pp. 186-194, 2002.

[14] I.-C. Wu, C.-L. Lai, S.-H. Bui Han et al., "Efficacy of entecavir in chronic hepatitis b patients with mildly elevated alanine aminotransferase and biopsy-proven histological damage," Hepatology, vol. 51, no. 4, pp. 1185-1189, 2010.

[15] R.-N. Chien, Y.-F. Liaw, and M. Atkins, "Pretherapy alanine transaminase level as a determinant for hepatitis B e antigen seroconversion during lamivudine therapy in patients with chronic hepatitis B," Hepatology, vol. 30, no. 3, pp. 770-774, 1999.

[16] L. Zhang, G. Wang, W. Hou, P. Li, A. Dulin, and H. L. Bonkovsky, "Contemporary clinical research of traditional chinese medicines for chronic hepatitis B in china: an analytical review," Hepatology, vol. 51, no. 2, pp. 690-698, 2010.

[17] S.-S. Dang, X.-L. Jia, P. Song et al., "Inhibitory effect of emodin and Astragalus polysaccharide on the replication of HBV," World Journal of Gastroenterology, vol. 15, no. 45, pp. 5669-5673, 2009.

[18] J.-S. Chang, H.-W. Liu, K.-C. Wang et al., "Ethanol extract of Polygonum cuspidatum inhibits hepatitis B virus in a stable HBV-producing cell line," Antiviral Research, vol. 66, no. 1, pp. 29-34, 2005.

[19] M. S. Shin, E. H. Kang, and Y. I. Lee, "A flavonoid from medicinal plants blocks hepatitis B virus-e antigen secretion in HBV-infected hepatocytes," Antiviral Research, vol. 67, no. 3, pp. 163-168, 2005.

[20] X. J. Zhu, X. H. Sun, L. Li et al., "Lingmao recipe inhibites reproduction of hepatitis B virus in vitro," Zhongxiyi Jiehe Ganbing Zazhi, vol. 26, no. 6, pp. 346-347 (Chinese).

[21] X. Y. Zheng, Guidelings For Clinical Research on Chinese New Herbal Medicines (Trial Implementation), Medical Science and Technology Publishing House of China, Beijing, China, 1993.

[22] J.-C. Xian, H.-T. Xu, Y.-L. He et al., "Liver histological changes in chronic hepatitis B patients with elevated ALT less than $2 \mathrm{x}$ ULN," Chinese Journal of Hepatology, vol. 19, no. 6, pp. 431-435, 2011 (Chinese).

[23] M. H. Nguyen, H. Trinh, R. T. Garcia et al., "Significant histologic disease in HBV infected patients with normal to minimally elevated ALT levels at initial evaluate-on (abstract)," Hepatology, vol. 42, supplement 1, p. 593A, 2005.

[24] S. Q. Liu, X. J. Zhu, X. H. Sun et al., "Characteristic of liver pathology in $\mathrm{HBeAg}$-positive and $\mathrm{HBeAg}$-negative chronic hepatitis B patients with mildly elevated AL-T,' Chinese Journal of Hepatology, vol. 20, no. 5, pp. 348-352, 2012 (Chinese).

[25] R. Kariv, M. Leshno, A. Beth-Or et al., "Re-evaluation of serum alanine aminotransferase upper normal limit and its modulating factors in a large-scale population study," Liver International, vol. 26, no. 4, pp. 445-450, 2006.

[26] H. C. Kim, C. M. Nam, S. H. Jee, K. H. Han, D. K. Oh, and I. Suh, "Normal serum aminotransferase concentration and risk of mortality from liver diseases: prospective cohort study," British Medical Journal, vol. 328, no. 7446, pp. 983-986, 2004.

[27] S. C. Li, S. C. Ong, S. G. Lim et al., "A cost comparison of management of chronic hepatitis B and its associated complications in Hong Kong and Singapore," Journal of Clinical Gastroenterology, vol. 38, no. 10, pp. S136-S143, 2004.

[28] D. J. Tenney, R. E. Rose, C. J. Baldick et al., "Long-term monitoring shows hepatitis $\mathrm{B}$ virus resistance to entecavir in nucleoside-naïve patients is rare through 5 years-of therapy," Hepatology, vol. 49, no. 5, pp. 1503-1514, 2009. 


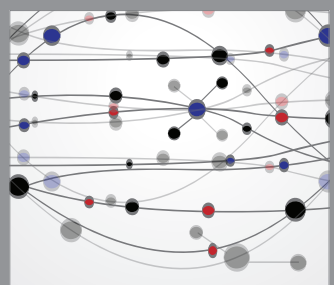

The Scientific World Journal
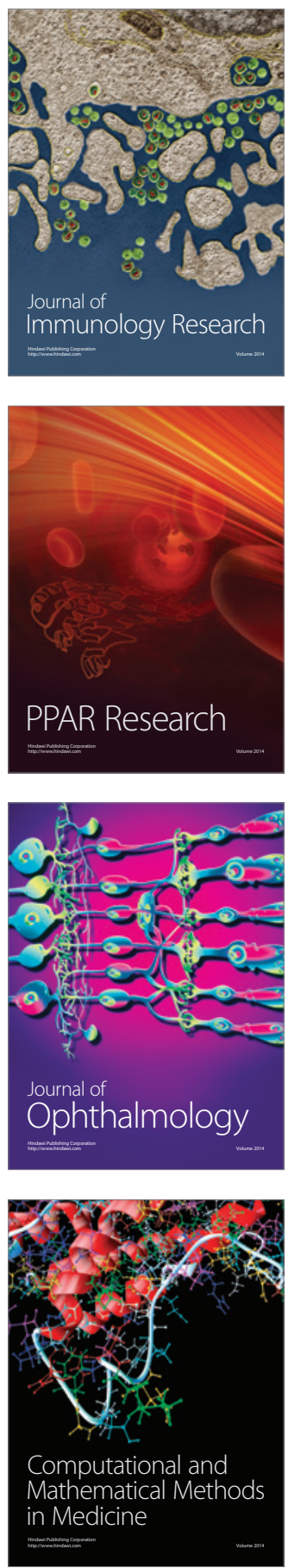

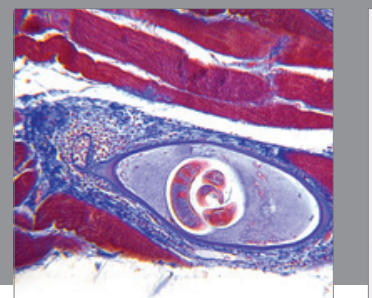

Gastroenterology

Research and Practice
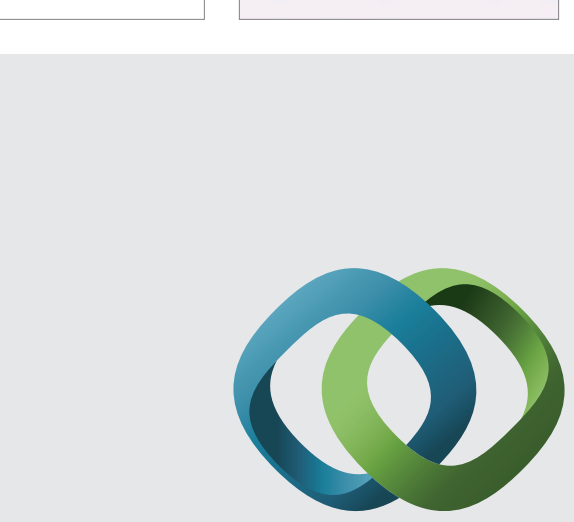

\section{Hindawi}

Submit your manuscripts at

http://www.hindawi.com
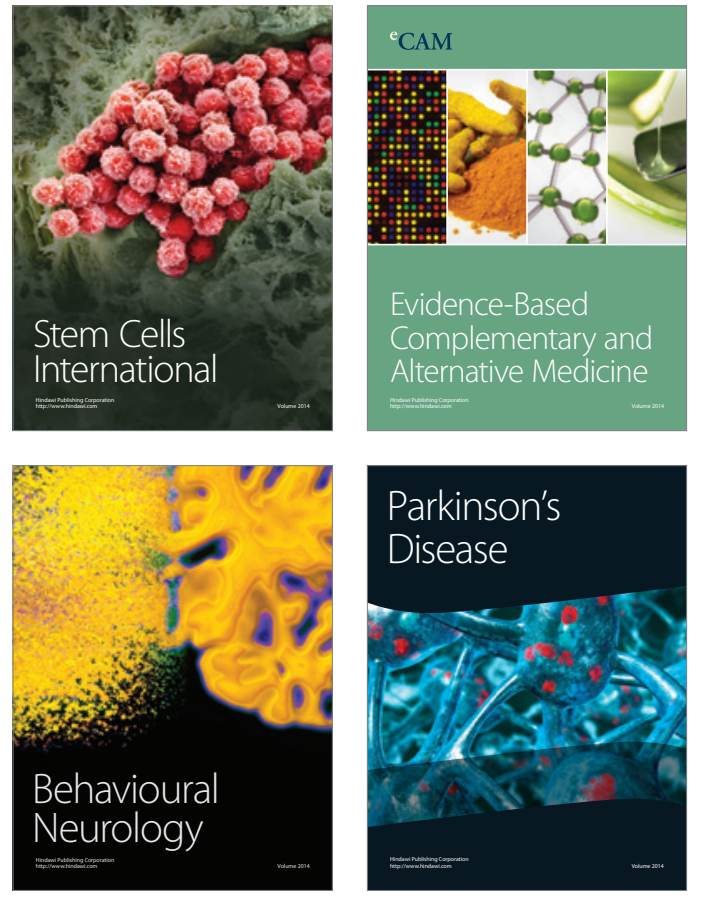
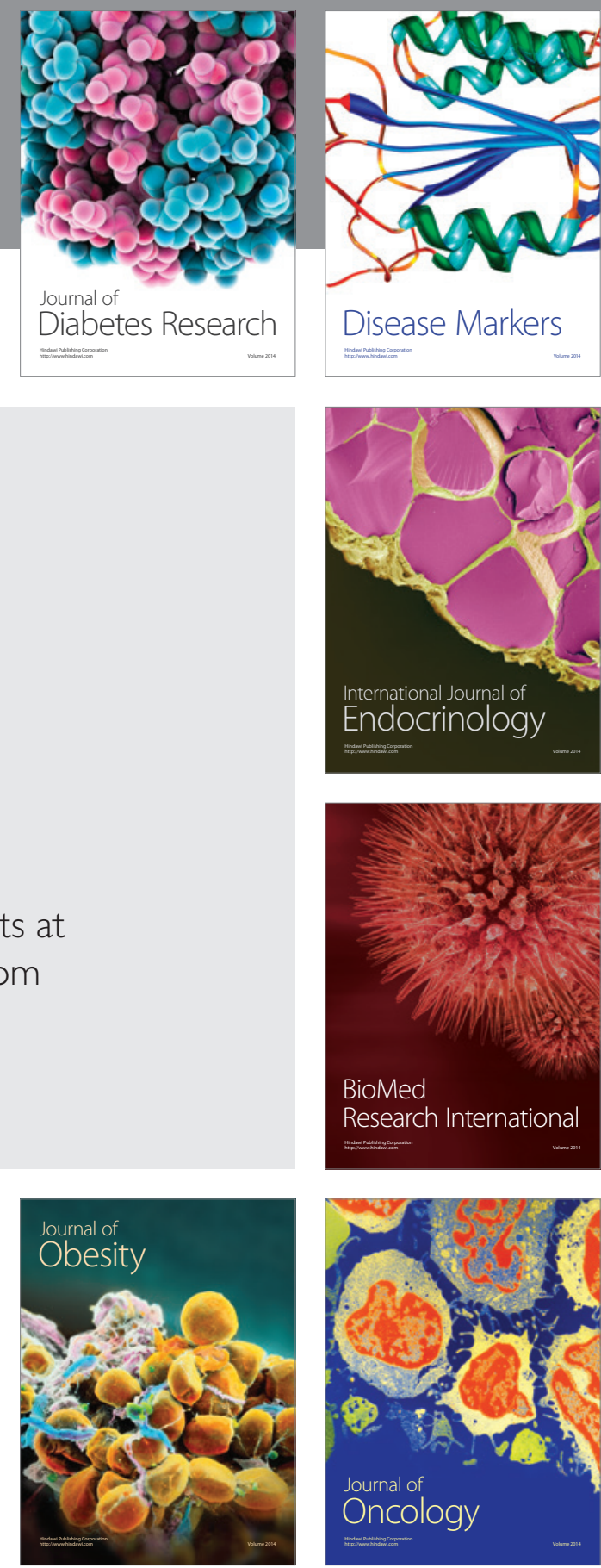

Disease Markers
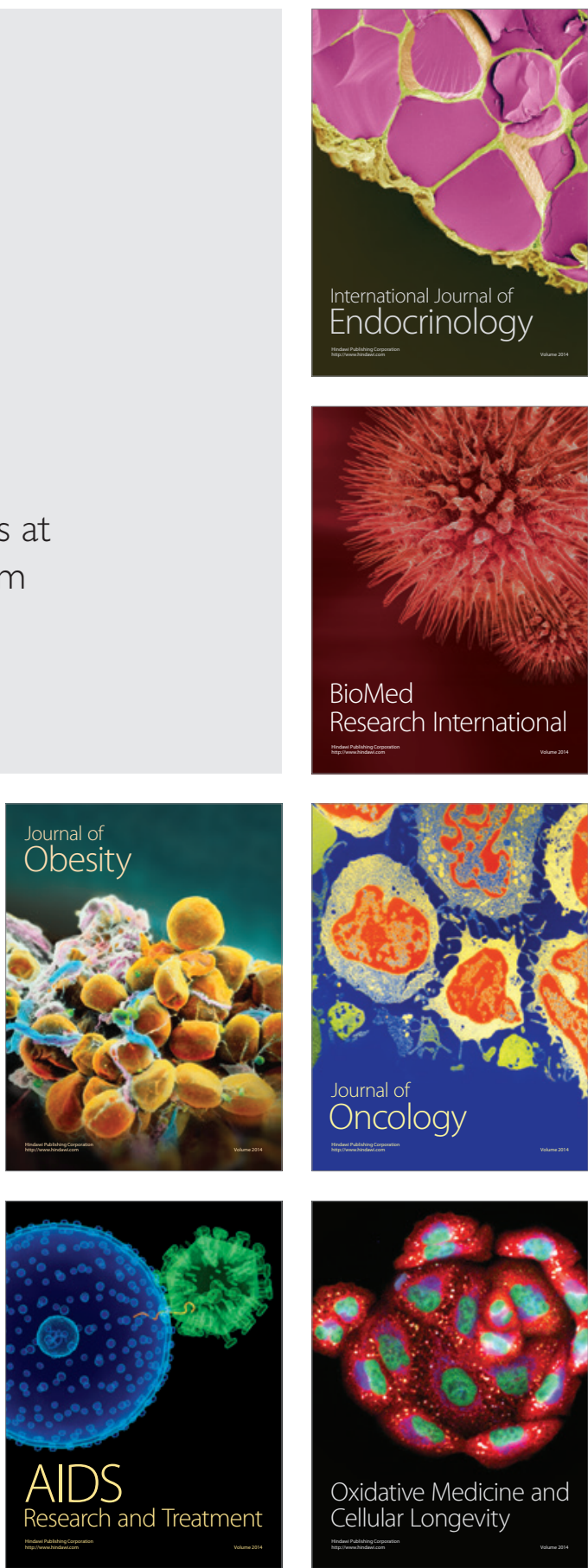